For The Epistemic Uses of Imagination, A. Kind and C. Badura (eds.), Routledge, forthcoming

**Please cite the published version**

\title{
8 Imagination, Inference, and Apriority
}

\author{
Antonella Mallozzi
}

\begin{abstract}
Is imagination a source of knowledge? Timothy Williamson has recently argued that our imaginative capacities can yield knowledge of a variety of matters, spanning from everyday practical matters to logic and set theory. Furthermore, imagination for Williamson plays a similar epistemic role in cognitive processes that we would traditionally classify as either a priori or $a$ posteriori, which he takes to indicate that the distinction itself is shallow and epistemologically fruitless. In this chapter, I aim to defend the a priori-a posteriori distinction from Williamson's challenge by questioning his account of imagination. I distinguish two notions of imagination at play in Williamson's account - sensory vs. belief-like imagination - and show that both face empirical and normative issues. Sensory imagination seems neither necessary nor sufficient for knowledge, whereas belief-like imagination isn't adequately disentangled from inference. Additionally, Williamson's examples are ad hoc and don't generalize. I conclude that Williamson's case against the a priori-a posteriori distinction is unconvincing, and so is the thesis that imagination is an epistemic source.
\end{abstract}

\section{Keywords}

Imagination; Inference; A Priori Knowledge; Williamson. 


\subsection{Introduction}

Is imagination a source of knowledge? Timothy Williamson has enthusiastically advocated the epistemic role of imagination for knowing a variety of matters $(2007,2013,2016)$. Its positive uses span from helping us plan and make choices in our life, to evaluating counterfactuals for various practical as well as philosophical purposes, to guiding us through knowledge of complex matters like logic and set theory. In core examples, we run cognitive processes "offline" in imagination by deploying helpful mental simulations. Imagination seems so powerful that Williamson conjectures it might have given us an evolutionary advantage as a species, as opposed to being a mere accidental byproduct of our evolutionary history. This fits an overall anti-exceptionalist program in epistemology, which urges us to avoid positing special faculties and instead look for explanations that are continuous with our ordinary and scientific knowledge of the world (2007).

Williamson's account of imagination also contributes to a critical task. He challenges the philosophical significance of the long-established distinction between a priori and a posteriori knowledge by appealing to a similar role that imagination seems to play in typical cases from each category. Williamson's argument proceeds by looking at examples of a priori and $a$ posteriori knowledge and comparing the sorts of cognitive processes we go through in each case, aiming to show that they are actually not that different. If that's correct, the distinction fails to capture anything epistemologically significant.

The primary aim of this chapter is to defend the a priori-a posteriori distinction from Williamson's challenge by questioning the claim that imagination is an epistemic source. I distinguish two notions of imagination at play in Williamson's account - sensory imagination and belief-like imagination - and show that both face empirical and normative issues. Sensory 
imagination seems in general neither necessary nor sufficient for knowledge; additionally, Williamson's particular examples are unconvincing because they don't generalize. Belief-like imagination, on the other hand, isn't sufficiently disentangled from inference. Overall, the $a$ priori-a posteriori distinction is still safe. Imagination seems at best to play only a supportive role for knowledge.

\subsection{Williamson's Challenge}

Williamson argues that the difference between a priori vs. a posteriori knowledge is only superficial. If we look carefully at the epistemic processes we carry out in clear cases from each category, those are apparently neither purely empirical nor purely a priori according to traditional standards. In particular, in many cases for Williamson experience plays a role that is neither strictly evidential, nor merely enabling for knowledge (2013: 13-4). The distinction is used in the epistemology of the a priori to answer the following worry. Even in apparently clearcut cases of a priori knowledge and justification, experience will be involved in enabling us to form the relevant judgments. Appeal to the distinction clarifies that experience is allowed to play this type of "enabling role" consistent with the knowledge in question remaining a priori, as long as it doesn't play an evidential role. In particular, experience might be enabling in the sense that we might only need it to initially acquire ingredient concepts of truths that we may then come to know a priori; or in the sense that it might enable our cognitive access to certain content. For example, one needs experience in order to acquire the concepts "red" and "green". However, that is merely enabling for one to come to know a priori that "An object cannot be red all over and green all over at the same time". Analogously, one needs to see the passages of a long mathematical proof on a piece of paper, or to memorize them, in order to carry out the proof. But seeing those passages, or recalling them in memory, isn't what justifies one's belief in the truth 
of the proof. Rather, those perceptual or mnemonic experiences only enable one to perform the relevant calculations and access the relevant mathematical propositions. Accordingly, coming to know the proof is still a priori. By contrast, when experience plays an evidential role, one's having certain experiences is what justifies the resulting judgments, so that those are a posteriori. For example, seeing or remembering the keys on the table constitutes evidential experience for knowing that the keys are on the table.

Williamson disagrees. He argues that in both apparently clear-cut cases of the a priori and the $a$ posteriori, respectively, experience plays a role that's intermediate between evidential and enabling. ${ }^{1}$ As we will see in a moment, the epistemic processes in question are distinctively imaginative. But it's important to stress that Williamson is not trying to reject the a priori as such or reduce all knowledge to the deliverances of our experience. Radical empiricism isn't an option, since experience might well be less than strictly evidential for knowledge. Nor should we conclude that such cases are "borderline"; in fact, he concedes that we can still identify examples from each category. The point is rather that the a priori-a posteriori distinction is a shallow one, which "does not cut at the epistemological joints" (2013: 8). That's a pragmatic moral: epistemologists would do better without these obsolete categories.

On the other hand, Williamson invites us to adopt a different framework, where imagination is a positive source of knowledge. For him, imagining has a key epistemic function in that we generally gain knowledge of various practical and theoretical matters by running our cognitive capacities “offline” via simulation in imagination. More precisely, from Williamson's discussion it appears that simulative imagination can be understood in two ways. The first, let's call it sensory imagination, is imagistic and involves simulation of our perceptual capacities. The second, let's call it belief-like imagination, is generally independent of the occurrence of mental 
imagery and involves simulation of our reasoning and planning capacities. Williamson's early examples against the a priori-a posteriori distinction $(2007,2013)$ feature the former "sensory" kind of imagination. We shall start with that. We will turn to belief-like imagination in Section 8.4 .

In core cases Williamson discusses, imaginative procedures are distinctively quasi-perceptual, namely they simulate regular perception (particularly, vision) offline. Since our judgments in the relevant cases are taken to result from such simulations in imagination, Williamson can then claim that the contribution of experience is neither strictly evidential, nor merely enabling for the resulting knowledge. The contribution of experience is rather intermediate, due to the quasiperceptual nature of the imaginative processes. Hence, even if by bottom-up standards we might still identify supposedly clear cases of the a priori-a posteriori, it turns out that the epistemic role of experience isn't straightforward. If Williamson is right, that directly undermines the significance of the a priori-a posteriori distinction.

Let us look at Williamson's core examples (2013):

(CR) All crimson things are red (WW) All recent copies of Who's Who are red

Williamson takes (CR) and (WW) to be clear examples of truths that, according to the tradition, one may come to know a priori and a posteriori respectively. Regarding (CR), the familiar idea is that one can come to know it by conceptual, or linguistic, analysis. "Red" may be understood as part of the concept "crimson"; or, by definition, "crimson" picks out a particular shade of red. On the other hand, (WW) is generally taken to be an example of a truth that we can only come to know through empirical means - plausibly, visual perception, or testimony. In order to know (WW), we need some direct or indirect experience with recent copies of Who's Who. However, 
Williamson disagrees with the traditional picture and offers a different story which distinctively features imagination of how one may come to know both of these truths.

As to (CR), he asks us to suppose that an agent, Norman, learns the words "red" and "crimson"

[...] independently of each other, by ostensive means. He learns 'crimson' by being shown samples to which it applies and samples to which it does not apply, and told which are which. He learns 'red' in a parallel but causally independent way. He is not taught any rule like [CR], connecting 'crimson' and 'red'. Through practice and feedback, he becomes very skilful in judging by eye whether something is crimson, and whether something is red. (9)

How does Norman come to know (CR)? Apparently, simply by imagining a shade of crimson and judging, within the imaginative supposition, that it is in fact red - without any further empirical support. The relevant epistemic process is described in the following passage, which is worth quoting at length:

First, Norman uses his skill in making visual judgments with 'crimson' to visually imagine a sample of crimson. Then he uses his skill in making visual judgments with 'red' to judge, within the imaginative supposition, 'It is red'. This involves a general human capacity to transpose 'online' cognitive skills originally developed in perception into corresponding 'offline' cognitive skills subsequently applied in imagination. [...] No episodic memories of prior experiences, for example of crimson things, play any role. As a result of the process, Norman accepts [CR]. Since his performance was sufficiently skillful, background conditions were normal, and so on, he thereby comes to know [CR]. 
Williamson's treatment of (WW) mirrors (CR). Norman has already learned "red". Now he learns all the other ingredient terms in (WW), i.e., "recent", "volume", etc., in a similar way. Through practice, he becomes similarly skillful at judging whether a certain volume is a copy of Who's Who. Then, without any further empirical investigation, but simply reflecting on what he has learned, Norman comes to know (WW) like he learned (CR). He visually imagines a recent volume of Who's Who, and “he uses his skill in making visual judgments with 'red' to judge, within the imaginative supposition, 'It is red"” (11).

Note that although those cases are thought-experiments, Norman's use of imagination in his epistemic performances isn't meant to be merely hypothetical. Nor is Norman special compared to other subjects. Instead, Norman's imaginative processes are supposedly representative of what epistemic subjects actually do. Being able to gain knowledge by running certain skills offline in imagination instantiates for Williamson a "general human capacity" (9).

Williamson's conclusion is straightforward. If typical cases of a priori and a posteriori knowledge involve similar cognitive processes or epistemic procedures, where experience contributes to an extent that is neither enabling nor evidential for the resulting knowledge, then the significance of the a priori-a posteriori distinction fades away.

\subsection{Issues for Sensory Imagination}

Williamson's challenge against the significance of the a priori-a posteriori distinction depends on making a convincing case that one may come to know truths from each category, such as (CR) and (WW), through similar imaginative processes. Specifically, imagination is here supposed to yield knowledge by deploying offline simulation of perceptual skills. Furthermore, the epistemic procedures featuring in Williamson's candidate examples shouldn't be special but 
generalizable to other truths and ways of knowing that are standardly regarded as a priori-a posteriori. Let us distinguish these two components:

Imagination Claim We may gain knowledge via sensory imagination by simulating perceptual cognitive skills offline.

Generality Claim Norman's epistemic procedures for knowing (CR) and (WW) are generalizable to standard cases of the a priori-a posteriori.

In Section 8.3.1, I discuss in detail the following three objections to the Imagination Claim (IC). First, although (IC) is an empirical thesis, it lacks adequate empirical support. Second, Williamson's treatment of (IC) suggests that simulative sensory imagination guarantees that Norman gains the relevant knowledge, based on considerations of reliability. However, not only is the reliability of sensory imagination quite speculative, but appealing to it also doesn't seem sufficient to establish that imagination is a source of knowledge. Third, (IC) prompts a dilemma concerning simulation, both horns of which have undesirable theoretical consequences. By undermining (IC), these criticisms undermine Williamson's case against the a priori-a posteriori distinction.

In Section 8.3.2, I argue that the Generality Claim (GC) is false. Even if (IC) were true and Norman came to know (CR) and (WW) in the described way, I argue that those examples are ad $h o c$ and don't generalize. Specifically, sensory imagination doesn't seem in general necessary for our epistemic processes. I conclude that the a priori-a posteriori distinction remains safe, whereas sensory imagination seems at best to only play a supportive role for knowledge. Finally, in Section 8.4, I turn to Williamson's more recent treatment of imagination as not essentially imagistic but rather "belief-like". I raise a general problem of clarifying the distinctive role of this type of imagination as opposed to inferential reasoning and contend that the latter best explains Williamson's further examples. 


\subsubsection{Against the Imagination Claim}

(a) An Empirical Matter

Williamson's (IC) is an empirical thesis. It concerns what epistemic subjects actually do, by pointing to the psychological processes that appear to be involved (according to Williamson's own introspective experience, at least) in coming to know truths such as (CR) and (WW). Those processes centrally involve visualizing "offline" percepts of the relevant shades of colors. More generally, imagination simulates cognitive skills that depend on perception.

Simulation has received considerable attention in the cognitive sciences as the main alternative against the received view for explaining certain cognitive capacities, especially practical ones. Roughly, the received view invokes an internally represented body of rules or propositions (possibly "tacit" or "sub-doxastic"), which guide a range of cognitive tasks. By contrast, simulation theory holds that certain mental mechanisms or components can be detached from their usual function and used to perform or support some other function offline (often via pretense). Simulation has been mainly thought to explain the capacity for "mind-reading" or attributing mental states and predicting other people's behavior without drawing from some underlying body of knowledge. But some maintain that similar offline processes might contribute to explain grammaticality judgments, empathy, counterfactual reasoning, and, importantly for our purposes, mental imagery (see Nichols et al. 1996 for an early survey). For example, Gregory Currie (1995) argues that episodes of imagery are episodes of simulated vision, based on evidence that they share central processing (same areas of brain involved, same content structure, similar incapacities from brain impairments). 
In his early examples involving (CR) and (WW), Williamson seems to think along similar lines as Currie. Imagination here simulates perception in a quasi-sensory way, by exploiting the same cognitive skills offline. Norman comes to know (CR) and (WW) through visual judgments within the imaginative supposition, which features offline simulation of visual experience. Additionally, for Williamson this hypothesis is supposed to explain even more abstract cases like knowledge of set theory and basic logical theorems, which are usually thought to be unrelated to sensory experience (and thus a priori). Williamson conjectures that we (well, mathematicians!) form conceptions of sets based on imaginative exercises that simulate "our online skill in observing and engaging in processes of physical creation" and remarks that perceiving such conceptions as "intuitively compelling" may rest on helpful mental "metaphors" and "pictures" that we develop in imagination (2013: 21-2). Similarly, in the case of knowledge of basic logical theorems, e.g., the reflexivity of identity $(\forall x x=x)$, imagination is alleged to play a key epistemic role by drawing from the experiential process of "continually judging numerical identity or distinctness among objects perceived or remembered in a wide variety of guises" (26). Williamson (2007) gives yet another example to the same effect: "If two marks had been nine inches apart, they would have been at least nineteen centimeters apart”. This judgment also supposedly depends on a simulative capacity of running naked eye measurements "visually offline" in imagination $(166) .^{2}$

Since (IC) concerns what epistemic subjects supposedly actually do, we would expect Williamson to support it with plenty of empirical evidence, like data from psychological or neuroscientific studies on actual subjects. It is thus surprising that he doesn't provide any such evidence for his claim, thus leaving it largely speculative. We saw that Williamson mentions a general human capacity for transposing perceptual cognitive skills into corresponding offline 
cognitive skills applied in imagination. Although that's broadly consistent with certain literature from the cognitive sciences, it is important to flag that simulation theory broadly construed is still contentious. Empirical data haven't clearly indicated that subjects actually deploy simulation in the relevant circumstances. For example, Nichols et al. (1996) assess empirical results concerning various applications of the theory and conclude that they are "still extremely sceptical of the off-line simulation theory of behavior prediction" (45) as well as "not sure how simulation is supposed to illuminate the issue of mental imagery" (35). (See also e.g. Gallagher (2007) and Barlassina and Gordon (2017) for evidence against mindreading in particular.) Additionally, researchers disagree as to the nature of simulation itself and its alleged contribution to our cognitive tasks. For example, Jane Heal remarks that "we have very little idea of what would be involved, neurophysiologically or functionally, in taking a system 'off-line'". What's worse, "we do not really know how to test simulationism, regarded as this empirical hypothesis" (1998: 89-90). Similarly, Shannon Spaulding warns us that the concept of simulation itself is problematic: "There is no consensus on what exactly simulation is in the cognitive sciences, not even in the area of mindreading" (2016b: 263). Insofar as the simulation hypothesis is still contentious and developing, (IC) rests on an uncertain basis. (Note that these considerations don't affect just sensory imagination qua simulating our perceptual processes, but also belief-like imagination, which I discuss below.)

Lacking support from the simulation hypothesis, Williamson's psychological remarks won't have their purported import. For it is not obvious that investigating the phenomenology of imagination per se would tell us anything relevant for epistemological purposes. The introspective appearance and contents of our conscious processes might tell us nothing much about their origin and justification. In particular, once divorced from the thesis that imagination 
and perception share central processing, the occurrence of mental imagery might be completely inessential to the cognitive tasks in question, or merely a contingent byproduct of the actual epistemic processes. I will return to the significance of this point.

\section{(b) Sensory Imagination and Reliability}

Appealing to simulation of perceptual capacities might help Williamson's case by suggesting that the imaginative procedures in question are reliable. Through offline visual simulation in imagination, Norman comes to know (CR) and (WW). I take Williamson's implicit assumption to be that since perception is a reliable source of knowledge, imagination qua offline perception must somehow "inherit" that reliability. By exploiting the same cognitive resources as perception, sensory imagination should be equally generally truth-conducive, to the effect that judgments reached within the imaginative supposition may constitute knowledge as well as in the corresponding online perceptual cases. Indeed, Norman seems guaranteed to reach the correct judgments based on the simulative processes' reliability. Williamson's claims presuppose an externalist conception of knowledge, where our epistemic states primarily depend on our relations with the external environment. Additionally, whether a subject knows (or is at least justified in believing) a certain truth seems to be a matter of the reliability of the relevant beliefforming processes, rather than one's reasons for believing that truth. Experiential processes like perceiving and remembering constitute sources of knowledge because they are generally truthconducive not because they provide evidence for believing their content. The thesis that Norman gains knowledge of (CR) and (WW) in the described way heavily relies on such externalist and reliabilist assumptions (similarly, Boghossian: Ch. 9 in Boghossian and Williamson 2020). 
But the idea that sensory imagination guarantees knowledge is problematic. To start, note that appealing to simulation per se doesn't ensure that this kind of imagination is generally truthconducive. Simulation might clarify how sensory imagination operates at the cognitive level, namely by sharing central processing with perception. But unless our imaginative exercises are suitably constrained, such that they consistently yield correct judgments, simulative imagination might still lead us astray. The scenarios we imagine depart from reality in all sorts of ways. Even among equally realistic scenarios, nothing in the imaginative exercise per se indicates which ones are correct. Indeed, by contrast with perception, our imaginative exercises do not generally entitle us to form beliefs based on the imagined content, like we do based on percepts. Candidate accounts of the epistemic uses of imagination should clarify under what conditions our imaginative exercises yield the correct outputs (similarly Kind and Kung 2016; Langland-Hassan 2016; Nichols 2006; Spaulding 2016a.).

Williamson seems to trust our imaginative capacities to be reliable somehow by default, because they simulate online skills that are themselves reliable. Norman might mistakenly visualize a peripheral shade of crimson, but "the relevant cognitive skills must include sensitivity to such issues" (2013: 10). Still, trusting imagination to be somehow reliable by default when deployed for certain cognitive tasks is far from establishing that it is a source of knowledge. Additionally, even granting that it is in fact reliable, that doesn't seem sufficient for knowledge. Norman's imaginative capacities might be perfectly reliable and still he may not have knowledge, since he might invariably be making merely lucky guesses. One should be able to provide reasons for taking the judgments resulting from our imaginative exercises to be correct.

The issue is symptomatic of a broader concern that Williamson's empirical hypothesis about the epistemic roles of imagination neglects central questions of normative epistemology. ${ }^{3}$ In order to 
establish that imagination is a source of knowledge, it is not sufficient to speculate about the psychological processes subjects go through when engaging in imaginative exercises and trust that these are reliable based on possibly sharing central processing. One should integrate an account of the specific constraints that bind our imaginative exercises and explain why they consistently deliver the correct results, while also ruling out that they are merely lucky guesses.

\section{(c) Simulation and Cognitive Architecture}

The simulation hypothesis at play in core examples (CR) and (WW) seems to commit one to a precise cognitive architecture. If our imaginative exercises simulate perceptual capacities offline, imagination and perception at least share the same cognitive mechanisms. Indeed, there might be just one faculty or cognitive unit, which can work in both online and offline modes. We saw that this might provide partial support for Williamson's thesis that imagination is a source of knowledge, at least granted that the offline operations of the unit are generally as truth-conducive as the online perceptual ones. However, on this supposition the alleged productive work of imagination remains unexplained.

In Williamson's examples, imagination seems to be doing more than just simulating perception offline. It does not merely reproduce one's perceptual experience, but is also responsible for filling a gap, so to speak, between what we experienced and what we have not in reasoning aimed at generalization. Imagination seems responsible for productive transitions beyond observed instances of a certain phenomenon. In the described procedure for knowing $(\mathrm{CR})$ and (WW), imagination yields the relevant generalizations by connecting and supplementing - in a Humean fashion, we might say - the limited data provided by perception. ${ }^{4}$ Through an imaginative procedure, Norman can somehow go beyond his repeated observations of shades of crimson as well as copies of Who's Who and reach general judgments. More generally, these 
sorts of transitions seem possible because of imagination, whereas perception alone does not suffice to move beyond the observed cases. Imagination has a central epistemic role by deploying this synthetic or productive capacity.

But note that there's nothing especially sensory at play in this alleged productive capacity. Indeed, this interpretation seems incompatible with understanding imagination as simulating perception. In simulation, sensory imagination runs our perceptual processes vicariously, by reproducing them offline. But it's not clear how such a cognitive unit can also yield generalizations like (CR) and (WW). If imagination does play that synthetic or productive role, imagination and perception might not share central processing after all; or, at least, they might not constitute one single faculty or cognitive unit but rather two distinct ones with different cognitive tasks. But this is an unwanted result for Williamson. If there is a separate faculty or cognitive unit of imagination, the thesis that our imaginative exercises ought to be explained in terms of perceptual simulation loses support. What is more, introducing such a special faculty or cognitive unit in order to explain the relevant ampliative work appears to clash with Williamson's anti-exceptionalism in epistemology, a view that requires that one avoid postulating sui generis epistemic faculties and that one seek to explain various types of knowledge by appealing to the same general human cognitive capacities.

Williamson seems to be facing a dilemma. Either (a), following the simulation hypothesis for sensory imagination, imagination and perception share the same cognitive mechanisms, which simplifies the cognitive architecture of imagination with all the annexed advantages that we saw. However, in this case we can't accommodate the supposed productive work of imagination. Imagination merely reproduces or copies what we have already experienced. Or (b), imagination and perception are separate and imagination is distinctively productive. However, that seems to 
give up the advantages that come from treating imagination as quasi-perceptual, particularly against the a priori-a posteriori distinction. Moreover, it may lead to positing some potentially exotic separate source of knowledge. From Williamson's discussion, it isn't clear that he could avoid the dilemma. There seems to be no third option that allows one to account for both the quasi-perceptual and the productive capacities that imagination seems to have in his examples.

To take stock, (IC) rests on shaky grounds. The claim lacks adequate empirical support, while it appears to assume that simulative sensory imagination somehow guarantees knowledge outputs. Additionally, the purported roles of sensory imagination should be clarified to avoid an unwanted dilemma for our cognitive architecture. Overall, more is needed to establish (IC) and thereby undermine the a priori-a posteriori distinction.

\subsubsection{Against the Generality Claim}

There is a deeper difficulty for Williamson's challenge against the a priori-a posteriori distinction: (GC) seems false. Even if we granted that (IC) were true and Norman came to know (CR) and (WW) in the described way, those epistemic procedures aren't generalizable to standard cases of the a priori-a posteriori. Hence, they won't manage to jeopardize the significance of the a priori-a posteriori distinction. ${ }^{5}$ More generally, sensory imagination doesn't seem necessary for knowledge.

Williamson remarks that imagination need not in general involve mental imagery (more below, Section 8.4). However, note that mental imagery is essential for Norman's coming to know (CR) and (WW). Norman needs to engage in imaginative processes that involve offline visual simulation in order to formulate the relevant judgments. That, in turn, is crucial for Williamson's dialectical purposes. Since offline simulation in imagination is only quasi-perceptual, experience appears to contribute to the resulting knowledge merely to an intermediate extent. More 
precisely, experience is supposedly neither strictly evidential nor merely enabling for coming to know (CR) and (WW). Thus, eliciting a familiar feeling of "experiencing" quasi-perceptual contents in imagination is crucial for Williamson's argument because that's how we would find support for the claim that imaginative processes straddle the traditional a priori-a posteriori categories. Indeed, those examples might feel intuitive, since they fit well the phenomenology of imagining based on simple introspection. However, I shall argue that the examples are devised to have sensory imagination play a crucial role and misleadingly induce us to think that they would generalize to similar cases. Additionally, there is a problem with the fact that the examples characteristically feature universal statements. That misleadingly induces us to think that imagination (this time not necessarily imagistic) is required to explain the productive epistemic process through which Norman reaches the universalizations. That's especially relevant in the case of (WW), so let's start with that.

Example (WW) seems a rather strange choice for an a posteriori truth. Why "All recent copies of Who's Who are red", rather than, simply, “This copy of Who's Who [here, in front of me] is red"? Ostensive, "here and now" judgments would more naturally come to mind as clear-cut cases of the a posteriori, likely because of their direct perceptual character. On the other hand, (WW) is an empirical generalization. According to Williamson's narrative, Norman sees a certain number of copies of Who's Who and concludes based on his imaginative assessment that all copies look alike. Why choose this example, rather than a simply ostensive one? Well, universalizations call for an explanation of the epistemic process that allows one to go beyond particular observations, and one might find imagination to be a promising candidate. As we saw in the previous section, imagination might be thought of as productive. But then (WW) seems ad $h o c$ and won't generalize. We are induced to think that imagination is crucially involved in 
coming to know (WW) qua a standard case of the a posteriori; whereas the pull of the example lies in the imagination's potential to justify knowledge of universal statements.

Could someone complain that generalizations constitute nearly all of empirical science? What better example of the a posteriori? To my mind, the objection backfires. First, (WW) is not a scientific generalization. Furthermore, pushing this line requires showing how the proposed account applies to real generalizations of science - those that actually matter to us. The imaginative procedure carried out by Norman in the case of (WW) doesn't seem to plausibly account for the way in which, say, a biologist may come to know that "African rhinos have two horns, while Indian rhinos have one horn". ${ }^{6}$ On the contrary, imagination seems completely inessential for gaining that knowledge, which is done instead via empirical observation and inference. Thus, the objection highlights that example (WW) does not generalize but is rather $a d$ hoc.

Turning specifically to sensory imagination, both (CR) and (WW) generally serve Williamson's argumentative purposes because they hinge on visualizing shades of red. But for (CR) that is crucial. For it suggests that even in standard cases of the a priori some experiential contribution that is more than merely enabling is required for knowledge. However, because of the key role played by color terms, this case seems ad hoc, as well. To illustrate, note that Williamson's argument wouldn't go through if the example involved terms from different domains. Consider a sentence that has the same logical structure as (CR), but whose ingredient terms won't obviously prompt perceptual associations as they belong to chief a priori categories - say, mathematical, or moral terms. Consider:

(EV) All even numbers are divisible by 2 
Suppose that Norman has learned the ingredient terms in (EV), "number", "division", "2", etc., in the usual way and has become similarly skillful at applying them. If one then asked Norman whether (EV) holds, it's implausible that this time he would engage in any imaginative exercise to formulate the relevant judgment. In particular, sensory imagination seems completely inessential for gaining knowledge. Using color terms is devised to prompt corresponding mental imagery in the reader, aiming to generate a sense that even traditionally a priori ways of knowing are actually quasi-perceptual. But the example does not generalize: (CR) is ad hoc, as well.

So we have a similar verdict with respect to (CR) as we reached with respect to (WW). The main takeaway is that both of these examples, by involving color terms and universal statements, are devised to induce us to think that imagination is crucially involved in coming to know those truths. But the examples don't generalize, so they fail to jeopardize the significance of the $a$ posteriori-a priori distinction.

So far in this section we have granted that Norman is as described and comes to know (CR) and (WW) like Williamson says. We granted in particular that although Norman is "very skillful" with the relevant terms, he's never connected them in the appropriate way. I'd like to conclude by flagging that Norman's coming to know (CR) and (WW) by sensory imagination is quite implausible. Once certain odd assumptions are removed, sensory imagination no longer seems necessary even in those cases.

Think of (CR). It seems implausible that someone who mastered the words "crimson" and "red" wouldn't be able to know that shades of crimson are shades of red without adverting to imagination. Based on his semantic competence, Norman should be able to come to know (CR) simply by reflecting on the ingredient terms and drawing the appropriate inference. In fact, (CR) 
seems a clear instance of an epistemic analytic truth (in Boghossian's 1996 terminology), namely a truth that can be known to be true only by virtue of grasping its meaning. ${ }^{7}$ If Norman truly has mastered the relevant terms, he would come to know (CR) without any contribution from experience, even imaginative experience, besides what's needed for acquiring them and enabling him to reason with them. Nor would he have to rely on visual simulations in imagination. While mental images of red might pop up in his mind as he assesses these matters, these images arguably won't play an epistemic role for the resulting knowledge. At most they play merely a supporting role. For those mental images won't add any further content to what Norman already knows from his semantic mastery. To add to this point, consider Aphantasiac Norman, who's like Norman in all respects except he suffers from a congenital inability to experience mental imagery. It seems implausible that, given that he can't deploy visual simulation in imagination, he wouldn't be able to come to know (CR). Hence, Norman could come to know (CR) purely $a$ priori by usual means.

Next consider (WW). Besides conceptual mastery, Norman arguably needs to further rely on specific memories as well as background empirical knowledge in order to know this truth. However, Williamson denies that episodic memory contributes to knowing (WW) and concludes that its status can't be obviously a posteriori. But that seems odd. Norman plausibly remembers one or more copies of Who's Who and puts those (episodic) memories to good use when drawing the generalization. Furthermore, Norman will need to draw from background empirical knowledge. Particularly, he'll need some basic grasp of the type-token relation. Norman needs to know (possibly implicitly) that volumes of a certain publication are all printed in copies which come in the same size, color, number of pages, etc. That also contributes to inferring from the observation of particular samples of Who's Who to the generalization (WW). Once the odd 
assumption that Norman doesn't rely on memory or other information is removed, sensory imagination seems again inessential for the relevant epistemic process. Norman could come to know (WW) a posteriori by usual means.

\subsection{Imagination vs. Inference}

Williamson's core examples tied the epistemic powers of imagination to offline simulation and mental imagery. That is the first notion of imagination at play in his account - what I called "sensory" imagination, as it involves simulating our perceptual capacities.

However, Williamson has more recently stressed that our imaginative processes need not involve mental imagery but can be expanded to cover other cases. For example, "imagining that there is a golden mountain in Austria [...] does not entail that one forms a mental image of a golden mountain in Austria" (2016: 15). Likewise, "a politician [who] is trying to work out what his core supporters would do at the next election if he voted for gun control [...] imagines their reactions, but doing so need not involve mental imagery" (9). In other words, Williamson now cashes out his account in terms of so-called propositional imagination. It is common in the literature to distinguish between sensory imagination on the one hand and imagining-that or propositional imagination on the other hand. The first involves consciously entertaining mental images. These are analogous to our percepts (not only visual) and have referential content, namely the objects they represent. Instead, imagining-that or propositional imagination is a propositional attitude with a content that can be evaluated as true or false, but possibly abstract and non-imagistic. Propositional imagination seems rather isolated from perceptual experience and more similar to conceptual thought. Several contemporary philosophers take imagination to be intrinsically quasi-perceptual (e.g., Balcerak Jackson 2016; Kind 2001; Kung 2010); but others contend that imaginative processes can be independent of the occurrence of mental 
images, namely propositional (e.g., Chalmers (2002); McGinn (2004); Yablo (1993).

Conversely, according to Arcangeli (2019), mental imagery might be independent of imaginative processes). Thought-experiments, and practical and counterfactual reasoning, for example, are often thought to be carried out via propositional imagination (Kind and Kung 2016; Nichols 2006).

Importantly, if imagination is propositional and so need not involve mental imagery, the simulation hypothesis is no longer strictly tied to perception. Instead, the idea now seems to be that in imagination we may simulate online belief or inference, which need not be perceptual (possibly akin to Currie and Ravenscroft's (2002) notion of "recreative" imagination, where imaginings are mental states that may mimic beliefs and decisions, inter alia). Leaving aside concerns tied to the empirical accuracy of this version of the simulation hypothesis, turning to propositional imagination might help expand the scope of Williamson's account. But it also raises a number of philosophical concerns.

First, some taxonomy would help understand better Williamson's desired notion. Hypothetical attitudes such as supposing, or conceiving, while obviously in the vicinity of imagining in the propositional sense might be distinct from it and have different phenomenology and different cognitive goals (Balcerak Jackson 2016). Elsewhere Williamson has argued against the epistemic powers of conceivability (particularly for modal knowledge: 2007: Ch.5). On the other hand, he often seems to use "imagining" and "supposing" interchangeably. So Williamson could offer us some useful clarification of those various notions.

Second, there is a more fundamental worry concerning the role of (propositional) imagination within the broader architecture of our cognitive functions. One might wonder in what sense the capacity at stake is supposedly still imaginative, when it no longer deploys quasi-perceptual 
imagining but it's rather akin to belief and reasoning. Simply put, how does the revised candidate account distinguish between imagination and inference? "Inference" is here meant to broadly denote reasoning with beliefs toward a certain judgment. More precisely, following a prominent characterization, inference is a transition or "movement of thought" from some beliefs to a conclusion, where the thinker takes the conclusion to be based on or supported by those beliefs (Boghossian 2014). One might suggest that the difference has to do with the hypothetical nature of imaginative thinking, and possibly a functional difference between imaginative representation vs. belief. But inference can certainly involve suppositions as well as beliefs. The question simply remerges at a more fine-grained level. Namely, what characterizes distinctively imaginative hypothetical thinking as opposed to inferential hypothetical thinking? Once imaginative thinking has been emptied of its sensory imagistic content, the suspicion is that we might be dealing with the very same mental operation, namely one where the thinker proceeds from a certain supposition to its consequences.

The issue is especially pressing if we consider Williamson's recent remark that principles of deductive logic supposedly shape rigorous imaginative procedures (2016). In developing conditional suppositions in imagination, according to Williamson we often follow roughly the logical deductive procedures of tree tableaux. For example, in imagining that there is a golden mountain in Austria, one does something formally similar to applying the first-order tableaux rule for the existential quantifier and developing the imaginative supposition via deduction (similarly for the rules for conjunction, disjunction, etc.). Williamson himself raises the worry that the analogy with tableaux procedures might not be strongest, since in imaginative exercises we do not normally look for contradictions. However, if correct, the general contribution of rigorous deductive logical reasoning may enhance the reliability of our imaginative procedures, 
given that logical rules are truth-preserving. But on the other hand, the analogy reinforces the worry that the procedures in question may no longer be imaginative in any clear sense. The general problem (surely not just for Williamson's account) is that while a notion of propositional imagination may help us build a more inclusive theory, it also calls for clarifying the principled distinction between imagining $v s$. inferring as hypothetical attitudes that similarly feature suppositions. Furthermore, the theory should say why we need to resort to this distinctive notion of imagination in order to explain the relevant epistemic processes, as opposed to hypothetical inference. In general, a candidate account should identify the limitations of an inferentialist theory as well as the theoretical advantages of appealing to imagination. Part of the pressure to clarify the distinctive explanatory work of propositional imagination compared to hypothetical inferential reasoning stems again from Williamson's own anti-exceptionalism. If hypothetical inferential reasoning effectively explains the relevant epistemic processes, we don't need to invoke any "extra" faculty of (propositional) imagination.

To illustrate, consider Williamson's recent claim that imagination yields knowledge of "mundane, widespread matters of immediate practical relevance" (2016: 1). For example, he describes a group of distant ancestors about to enter an unknown forest. What sorts of epistemic and practical advantages might imagination yield? An "obvious answer" for him is that

An imagination will alert them to various potential dangers and opportunities [...] They imagine wolves in the forest; warned of the danger, they keep a sharper look-out for signs of wolves. They imagine edible berries in the forest; alerted to the potential opportunity, they look about for bushes of the right kind. In both cases, their imagination enables them to prepare for practically relevant possibilities, helping them avoid dangers and take advantage of opportunities. (3) 
But in what way is the episode described a case of "knowledge by imagination"? More precisely, how is imagination playing an epistemic role? And how does suddenly realizing that one might be surrounded by various dangers or opportunities require the work of imagination? In fact, nothing especially imaginative seems to be at play as those ancestors go explore that forest and make the relevant decisions. A more natural reading of Williamson's story sees the ancestors assessing various possibilities concerning their environment, thus reasoning hypothetically based on certain premises. Those premises implicitly or explicitly convey information based on the ancestors' own past experience (e.g., episodic memories of similar forests they may have previously explored) and/or analogous testimony from others. Such information is put to use in hypothetical reasoning, drawing a conditional conclusion. Something along the lines of: "If present unknown forest $y$ is sufficiently similar to previously experienced forest $x$, then there might be wolves and berries in forest y". Clearly the inference need not be explicit. Appealing to simple inference seems to be all that is needed in order to explain how our characters may reach some (conditional) empirical knowledge; whereas it is not clear why one should invoke imagination.

Furthermore, for Williamson this is an "involuntary" manifestation of the imagination, by "breaking into the stream of consciousness with reminders of dangers and opportunities" (4). $\mathrm{He}$ distinguishes that from a "voluntary" mode, where imagination can instead be "turned on" when needed for problem-solving purposes. However, Williamson also claims that imagination constitutes a reliable method for forming true beliefs, and hence yields knowledge, only in the voluntary mode not the involuntary one (7). In sum, it is unclear that imagination plays the required epistemic role for knowledge in such cases, that anything more than inferential reasoning is involved. 
Perhaps all Williamson wants imagination to contribute in the story is mental imagery - thus going back to the first, sensory type of imagination. The ancestors might be visualizing wolves and berries as they assess those matters. If that's the point, however, he should clarify why the occurrence of such images should play such a crucial epistemic role, as opposed to only being an accidental byproduct of inferring based on background knowledge. Plausibly, our ancestors would be able to reach the same conclusions regarding their surroundings without any imagery occurring, since those images won't add any further content to the ingredient beliefs in the ancestors' inferences. More generally, while the occurrence of mental images might help some subjects carry out various cognitive tasks, it seems contingent and inessential for the resulting judgments. One might well work one's way through understanding and learning theoretical as well as practical matters without virtually any images occurring. Accordingly, it seems fruitful to think of mental imagery rather in terms of a cognitive tool or heuristic, playing a possibly supportive but generally inessential role for knowledge. Williamson's own examples involving logic and set theory may fit well this "instrumental" account of imagination, since mental pictures and metaphors seem mostly an aid to understanding and reasoning. However, note that adopting the instrumental view would require some considerable adjustment to the account. Specifically, it would require introducing some principled criterion for disentangling cases where sensory imagination is supposedly epistemically necessary for knowledge - like in the original examples (CR) and (WW) - from cases where it is merely instrumental and thus neither necessary nor sufficient for knowledge. Importantly, if imagination is a tool or heuristic, it is not a source of knowledge.

Are things any different in cases where imagination supposedly operates in a voluntary mode? Perhaps its epistemic role is more straightforward in such cases. Consider Williamson's example 
of a hunter who is pondering whether to jump a stream that obstructs his way. The decision is "vital", since the hunter might die if he tries and fails. Williamson himself acknowledges that the hunter might easily imagine himself failing as well as succeeding in jumping the stream. However, "imagining oneself trying" somehow constitutes a reliable method for forming true beliefs. "Indeed [...] under suitable conditions, the method enables one to know what would happen in the hypothetical circumstances" (8). Williamson is confident that since this is a voluntary use of imagination, that ensures its reliability (7). This optimism is hard to share however, especially since he admits that "hardly anything has been said so far to explain the method's reliability" (8). Falling short of standards we usually take as basic in epistemology, it seems quite doubtful that the imaginative exercise that the hunter carries out yields knowledge. Indeed, his decision whether or not to jump shouldn't be based on that simulation. Perhaps, like the hunter, we would all imagine ourselves jumping in the described scenario. Perhaps that might sometimes help our overall assessment of the situation as we make the decision. But that isn't tantamount to imagination yielding knowledge. For the described imaginative processes might feel familiar, and possibly have heuristic roles, while being again only an accidental byproduct of the actual epistemic process.

An alternative account of the hunter's decision-making process agrees with Williamson that the hunter carries out conditional reasoning via supposition but contends that its epistemic value is independent of the occurrence of any imaginative exercise. Although imagining oneself jumping might perhaps contribute to improve the hunter's actual performance, all we need to explain his decision-making process is hypothetical inference based on empirical evidence. That includes evidence collected through perception (e.g., measuring by eye the width of the stream, estimating its depth, checking how strong the water stream is), introspection (evaluating how tired one is, 
whether one feels in shape or not, etc.), and empirical background knowledge (about mountain streams, rocks, etc., as well as episodic memories of similar experiences). The hunter decides (and rightly so) whether to jump based on the outcome of inferring from such empirical information, which likely happens largely implicitly and quickly. Since the evidence is perceptual, introspective, and mnemonic, the inference is clearly a posteriori.

In conclusion, in order to defend the epistemic powers of imagination qua propositional imagination, one needs to articulate criteria in virtue of which certain processes and states are distinctively imaginative as opposed to broadly inferential. Based on that, one should further make the case that explaining the relevant epistemic processes requires appealing to imagination. Or, short of that, one should make the case that an account that rests on the epistemic roles of imagination offers substantive theoretical advantages over an account that rests on hypothetical inferential reasoning. ${ }^{8}$ 


\section{References}

Arcangeli, M. (2019). The Two Faces of Mental Imagery. Philosophy and Phenomenological Research. https://doi.org/10.1111/phpr.12589

Balcerak Jackson, M. (2016). On the Epistemic Value of Imagining, Supposing, and Conceiving. In A. Kind and P. Kung (Eds.), Knowledge Through Imagination (pp. 41-60). Oxford: Oxford University Press.

Barlassina, L., and Gordon, R. (2017). Folk Psychology as Mental Simulation. The Stanford Encyclopedia of Philosophy. https://plato.stanford.edu/archives/sum2017/entries/folkpsychsimulation/

Boghossian, P. (1996). Analyticity Reconsidered. Noûs 30: 360-91.

- (2014). What Is Inference? Philosophical Studies 169: 1-18.

Boghossian, P., and Williamson, T. (2020). Debating the A Priori. Oxford: Oxford University Press.

Casullo, A. (2013). Articulating the A Priori-A Posteriori Distinction. In A. Casullo and J. Thurow (Eds.), The A Priori in Philosophy (pp. 249-74). Oxford: Oxford University Press. Chalmers, D. (2002). Does Conceivability Entail Possibility? In T. Gendler and J. Hawthorne (Eds.), Conceivability and Possibility (pp. 145-201). Oxford: Oxford University Press.

Currie, G. (1995). Visual Imagery as the Simulation of Vision. Mind \& Language, 10: 25-44. Currie, G., and Ravenscroft, I. (2002). Recreative Minds. Oxford: Oxford University Press. Gallagher, S. (2007). Simulation Trouble. Social Neuroscience 2: 353-65.

Heal, J. (1998). Understanding Other Minds from the Inside. In A. O'Hear (Ed.), Contemporary Issues in the Philosophy of Mind (pp. 83-100). Cambridge: Cambridge University Press.

Jenkins, C. (2008). Modal Knowledge, Counterfactual Knowledge and the Role of Experience.

The Philosophical Quarterly 58: 693-701.

Jenkins, C., and Kasaki, M. (2014). The Traditional Conception of the A Priori. Synthese https://doi.org/10.1007/s11229-013-0394-X

Kind, A., and Kung, P. (2016). Knowledge Through Imagination. Oxford: Oxford University Press.

Kind, A. (2001). Putting the Image Back in Imagination. Philosophy and Phenomenological Research 62(1): 85-109 
Kind, A. (2020). What Imagination Teaches. In John Schwenkler and Enoch Lambert (Eds.), Becoming Someone New: Essays on Transformative Experience, Choice and Change (pp. 133-46). Oxford: Oxford University Press.

Kung, P. (2010). Imagining as a Guide to Possibility. Philosophy and Phenomenological Research, 81, 620-663.

Langland-Hassan, P. (2016). On Choosing What to Imagine. In A. Kind and P. Kung (Eds.), Knowledge Through Imagination (pp. 61-84). Oxford: Oxford University Press.Mallozzi, A. (2020). Superexplanations for Counterfactual Knowledge. Philosophical Studies https://doi.org/10.1007/s11098-020-01477-0

Malmgren, A. (2011). Rationalism and the Content of Intuitive Judgements. Mind 120: 263-327. McGinn, C. (2004). Mindsight: Image, Dream, Meaning. Cambridge, MA: Harvard University Press.

Nichols, S. (2006). The Architecture of Imagination. Oxford: Oxford University Press.

Nichols, S., Stich, S., Leslie, A., and Klein, D. (1996). Varieties of Offline-Simulation. Theories of Theories of Mind 24: 39-74.

Quine, W.V.O. (1951). Two Dogmas of Empiricism. The Philosophical Review 60: 20-43. Spaulding, S. (2016a). Imagination through Knowledge. In A. Kind and P. Kung (Eds.), Knowledge Through Imagination (pp. 207-26). Oxford: Oxford University Press.

—. (2016b). Simulation Theory. In A. Kind (Ed.), The Routledge Handbook of Philosophy of Imagination (pp. 262-73). New York: Routledge.

Williamson, T. (2007). The Philosophy of Philosophy. Oxford: Blackwell.

—. (2013). How Deep Is the Distinction between A Priori and A Posteriori Knowledge? In A. Casullo and J. Thurow (Eds.), The A Priori in Philosophy (pp. 291-313). Oxford: Oxford University Press.

—. (2016). Knowing by Imagining. In A. Kind and P. Kung (Eds.), Knowledge Through Imagination (pp. 112-23). Oxford: Oxford University Press.

Yablo, S. (1993). Is Conceivability a Guide to Possibility? Philosophy and Phenomenological Research 53, 1-42.

\footnotetext{
${ }^{1}$ With this criticism Williamson challenges the a priori-a posteriori distinction as introduced by "bottom-up" standards, namely by examples. Williamson further criticizes "top-down" characterizations (namely, via definition), aiming to show that those are also epistemologically shallow. Since the latter criticism doesn't involve imagination,
} 
I won't discuss it here. But see Boghossian's reply to Williamson for an illuminating discussion of the issues involved with top-down characterizations (Boghossian and Williamson 2020: Ch. 9).

${ }^{2}$ For discussion, see Casullo (2013) and Jenkins (2008).

${ }^{3}$ This problem is especially pressing for Williamson's counterfactual account of modal knowledge (see Mallozzi 2020).

${ }^{4}$ Jenkins and Kasaki (2014) draw a similar parallel with Kant's imagination. Kind (2020) similarly calls this imaginative operation "scaffolding".

${ }^{5}$ Jenkins and Kasaki (2014) similarly complain that Williamson's examples are "too easy". Malmgren (2011) targets the 2007 example mentioned above, 'If two marks had been nine inches apart, they would have been at least nineteen centimeters apart'. She stresses that this is a peculiar case and it is implausible that it generalizes in the way Williamson wants.

${ }^{6}$ Thanks to Michael Devitt for emphasizing this point.

${ }^{7}$ Boghossian contrasts epistemic analyticity with metaphysical analyticity, i.e., the idea that certain truths are true only by virtue of meaning. According to him, Quine's arguments successfully rejected metaphysical analyticity, whereas epistemic analyticity can be salvaged (1996).

${ }^{8}$ Thanks to Chris Badura, Paul Boghossian, Michael Devitt, Amy Kind, David Papineau, and an anonymous referee for helpful comments on a previous draft. 